\title{
Aprendizaje mediante Juegos basados en Principios de Gamificación en Instituciones de Educación Superior
}

\author{
Ingrid A. Hernández-Horta*, Anderson Monroy-Reza y Martha Jiménez-García \\ Instituto Politécnico Nacional, Unidad Profesional Interdisciplinaria de Ingeniería y Ciencias Sociales y \\ Administrativas, Av. Té 950, Iztacalco, Granjas México, 08400 Ciudad de México. \\ (email: ingrid.hdz.horta@gmail.com; amonroyr1301@alumno.ipn.mx; majimenez@ipn.mx)
}

${ }^{*}$ Autor a quien debe ser dirigida la correspondencia

Recibido Ene. 29, 2018; Aceptado Abr. 2, 2018; Versión final May. 30, 2018, Publicado Oct. 2018

\begin{abstract}
Resumen
El objetivo de esta investigación fue diseñar una propuesta de elaboración de un juego basado en principios de Gamificación para Instituciones de Educación Superior (IES) en la Ciudad de México. El objetivo de esto es elevar el rendimiento académico de los estudiantes y complementar los procesos de enseñanza tradicionales. Se llevó a cabo una investigación teórica para encontrar los puntos más importantes de la Gamificación en la educación superior. Como resultados se tiene la propuesta de elaboración de un juego, que puede utilizarse en dispositivos móviles. Dicha propuesta integra componentes de juego, así como mecánicas o reglas de juego. Se concluye que la gamificación es una estrategia que toda IES debe implementar.
\end{abstract}

Palabras clave: gamificación; dispositivos móviles; juego; rendimiento académico; educación superior

\section{Learning through Games based on Principles of Gamification in Higher Education Institutions}

\begin{abstract}
The aim of this research was to design a proposal to elaborate a game based on the principles of Gamification for Higher Education Institutions (IES) in Mexico City. This was done with the aim of increasing the academic performance of students and complementing traditional education processes. A theoretical research was performed to find the most important points of gamification in higher education. As a result, a game that can be used on mobile devices was proposed. This proposal integrates game components, as well as the mechanics of the game or game rules. It is concluded that gamification is a strategy that all IES should implement.
\end{abstract}

Keywords: gamification; mobile devices; game; academic performance; higher education 


\section{INTRODUCCIÓN}

En el año 1994 México ingresó a la Organización para la Cooperación y Desarrollo Económico (OCDE), inmediatamente después de su incorporación, México tuvo que ajustar paulatinamente sus procesos educativos a propuestas creadas previamente por la OCDE para todos sus miembros; dichas propuestas dieron un nuevo enfoque a la educación, el enfoque de competencias; en este nuevo enfoque de la educación se busca desarrollar tres habilidades en los estudiantes las cuales son 1) saber, 2) saber hacer, y 3) querer hacer; esto con la finalidad que los estudiantes puedan ser capaces de afrontar y solucionar nuevos retos de forma adecuada y que aprendan de forma autónoma el resto de su vida (Gimeno, 2008). Las habilidades mencionadas con anterioridad son elementos básicos deseables para la obtención de competencias básicas. Una competencia básica puede catalogarse como tal si: 1) contribuye a la obtener resultados con gran valor personal o social, 2) Puede aplicarse a muchos contextos, y 3) Permite superar pruebas complejas de diversos ámbitos (Consejo Mexicano de Investigación Educativa y Moreno, 2015). Si bien uno de los propósitos de este nuevo enfoque educativo es la generación de competencias, también lo es el incrementar los niveles de compromiso del alumno con su aprendizaje (Moreno, 2012). Es por ello que dentro del aprendizaje basado en competencias se busca que el estudiante tenga más participación en sus procesos formativos, es decir que el alumno sea director y participe de su propia evolución educativa y que su aprendizaje sea continuo en todas las actividades que desarrolle.

Al buscar que los alumnos desarrollen competencias se han creado métodos de evaluación y desarrollo de competencias; dichos métodos persiguen los propósitos de integración de sistemas de evaluación innovadores y alternativos que se adapten a los cambios que la sociedad presenta (López et al., 2016). Algunos de los métodos son citados por Moreno (2012), quien en su investigación de evaluación por competencias, indica que los métodos más habituales para la evaluación y desarrollo de competencias son; 1) Observación, 2) Entrevista, 3) Proyectos, 4) Aprendizaje basado en problemas, 5) Estudio de caso, 6) Simulaciones y 7) Aprendizaje cooperativo. En el contexto de generación de competencias, integración de sistemas innovadores para la evaluación de las mismas, y el incremento del compromiso de los alumnos con su aprendizaje, es donde la Gamificación toma lugar como estrategia. La Gamificación es una técnica que permite el uso de los elementos de videojuegos, como lo son: los componentes, las mecánicas, y las dinámicas en entornos no lúdicos (Deterding Dixon et al., 2011); los componentes más comunes en entornos de Gamificación son: 1) Insignias, 2) Barras de nivel, y 3) Tableros de calificación (Zichermann y Linder, 2010); el uso de la técnica de gamificación se justifica por los beneficios que ésta propicia; dicha técnica es capaz de crear, fomentar, cambiar actitudes o comportamientos en los individuos involucrados a través del otorgamiento de refuerzos intrínsecos y extrínsecos (Deterding Sicart et al., 2011) lo cual añade valor al proceso en el cual ésta técnica este siendo involucrada.

Existen muchas organizaciones de diferentes giros, las cuales utilizan la técnica de Gamificación para mantener a la motivación de sus integrantes en los niveles más altos (Robson et al., 2015); la empresa Forsquare fue el primer producto en el rubro de desarrollo de software que hizo uso de la técnica de gamificación para lograr posicionarse con éxito en el mercado (Deterding Dixon et al., 2011); derivado del éxito que Forsquare obtuvo al implementar la gamificación, varias empresas se dedicaron al estudio y la implementación de la gamificación en sus productos y servicios, con el objetivo en particular de incrementar el compromiso y la lealtad por parte de sus consumidores y trabajadores (Zichermann y Cunningham, 2011); algunos ejemplos de productos que también han hecho uso de exitoso de la gamificación en sus aplicaciones móviles son 1) Software de AppleWatch, 2) Software Nike+, 3) Navegador Waze, 4) Gestor de reservas Booking, y 5) Sistema de Transporte Uber; en particular estas aplicaciones móviles han logrado crear y mantener un nuevo hábito es sus consumidores añadiendo valor a los productos y servicios que ofertan a través del uso de la técnica de gamificación. La técnica de Gamificación no es exclusiva del sector empresarial o de mercadotecnia, esta técnica también es aplicable en otros ámbitos. En el ámbito educativo, la Gamificación cuenta con una rama dedicada al estudio del uso de los componentes de juego dentro de los procesos de aprendizaje, dicha rama es llamada "Game Based Learning" es decir el aprendizaje basado en juegos, esta rama permite al estudiante poner a prueba su retención y su conocimiento, a través de actividades más dinámicas y desafiantes que las que actualmente son utilizadas en la educación tradicional ofertada (Khan et al., 2017).

Acorde con Brull (2016), el aprendizaje basado en juegos permite que los alumnos participen y creen una comunidad de aprendizaje, disfrutando de la libertad de experimentar y fallar en un entorno agradable. Así mismo los alumnos tienen la oportunidad de interactuar con sensaciones diferentes que los mantienen motivados. Existe evidencia de que los alumnos involucrados en entornos con Gamificación mejoran su aprendizaje, e incrementan su motivación y compromiso (Chu y Hung, 2015); en Estados Unidos de América, se han aplicado los elementos de la Gamificación en niveles de primaria y preparatoria y estos han propiciado un incremento en la capacidad retención de los alumnos y en el compromiso por parte de los mismos (Brull y 
Finlayson, 2016); así mismo dicha técnica también ha sido aplicada con éxito en niveles de secundaria ayudando a mejorar las calificaciones en las pruebas o exámenes de los alumnos involucrados (Admiraal et al., 2014).

Sin embargo, los alcances de la Gamificación no se enfocan únicamente en la educación básica, existen universidades que han aplicado con éxito técnicas de Gamificación como refuerzo a la educación tradicional, en donde se ha mostrado un incremento en la motivación de los alumnos y en su participación (Alhammad y Moreno, 2018; Çakıroğlu et al., 2017; Ebner y Holzinger, 2007). Una de las razones de que la Gamificación proporcione grandes beneficios en los alumnos, es el alto grado de aceptación por parte de los alumnos, la aceptación de la Gamificación ha mostrado una tendencia positiva, si el juego es diseñado de manera adecuada (Aldemir Celik y Kaplan, 2018). Por lo tanto, sugerir un concepto de diseño con juegos es un gran paso para mejorar la experiencia de aprendizaje en próximas generaciones (Lee y Doh, 2012).

A pesar de los beneficios que el aprendizaje basado en juegos aporta a los involucrados, esta técnica ha sido duramente criticada, puesto que existe el mito de que aprender y jugar no son términos que vayan de la mano; sin embargo, uno de los puntos a favor más significativos de ésta técnica es que se acopla a las demandas de nuevas generaciones, cuya vida está en constante interacción con las Tecnologías de Información y Comunicación (TIC) (Sanmugam et al, 2016); de hecho, uno de los mayores atractivos del aprendizaje basado en juegos es el uso de las TIC, las cuales son uno de los principales motivadores para el alumnado, durante los procesos del aprendizaje (García et al., 2014); por ello el correcto uso de los elementos de Gamificación, aunado al uso de las TIC es esencial para mantener la motivación en cada curso en el que se desee incorporar un sistema de Gamificación (Landers et al., 2017).

En esta breve reseña se han manifestado los propósitos tanto de la Gamificación como de los Modelos educativos por competencias, resumiendo tenemos, por un lado, a la Gamificación la cual busca crear y modificar actitudes y aptitudes dentro de los individuos involucrados en el juego; mientras que, por otro lado, los modelos educativos basados en competencias buscan que los alumnos desarrollen ciertas actitudes y aptitudes en específico. Derivado de la afirmación anterior se puede inferir que la Gamificación es un excelente auxiliar para modificar las actitudes de los estudiantes pertenecientes al modelo de aprendizaje por competencias, brindándoles una herramienta divertida, e innovadora para el desarrollo y evaluación de sus competencias a través de retos, misiones, y más elementos de juego.

Es por ello que el objetivo de esta investigación es diseñar una propuesta de elaboración de un juego basado en principios de Gamificación para Instituciones de Educación Superior (IES) en la Ciudad de México el cual persiga los propósitos de desarrollar competencias en los estudiantes y complementar los procesos de enseñanza tradicionales.

\section{MATERIALES Y MÉTODOS}

Existen más de quince metodologías diferentes para la correcta inclusión de la Gamificación y del buen diseño del juego, una de ella es la metodología 6D de Werbach y Hunter, estos autores desarrollaron su propuesta metodológica de inclusión y diseño de Gamificación a través de la observación de los juegos cotidianos, y su réplica en otros contextos, en especial en el de las organizaciones.

La propuesta de Werbach y Hunter, sugiere que; para poder crear un juego exitoso, se deben introducir tres elementos fundamentales de juego dentro del mismo, estos elementos son: 1) Componentes de juego, 2) Mecánicas y 3) Dinámicas; Sin embargo, antes de elegir dichos elementos de juego deben de seguir seis pasos los cuales se enlistan a continuación: 1) Definir objetivos del negocio, 2) Definir comportamientos deseados en el mercado meta, 3) Describir el tipo de jugadores hacia los cuales va dirigido el juego, 4) Elegir Actividades a realizar, 5) Incluir diversión en las actividades, y 6) Desarrollar herramientas (Werbach y Hunter, 2012). Gracias a su estructura, esta metodología flexible y se puede acoplar en casi cualquier contexto, inclusive hay investigaciones que utilizan esta metodología como un apoyo para el diseño de sistemas de Gamificación en la educación.

Esta investigación se valió del uso de la metodología de Werbach y Hunter, para el desarrollo de una propuesta de juego exitoso. Sin embargo, no se incluyeron los seis pasos propuestos por dichos autores, se replanteo la metodología de forma que solo se usaron cinco de sus pasos, mismos que se adaptaron al contexto estudiantil. Así mismo se adaptó la metodología acorde a las sugerencias de sus creadores en dos fases o pasos. En la Fig. 1 se pueden apreciar gráficamente los pasos que integraron a la metodología y las etapas correspondientes a cada uno de estos pasos (Ver Fig. 1). 


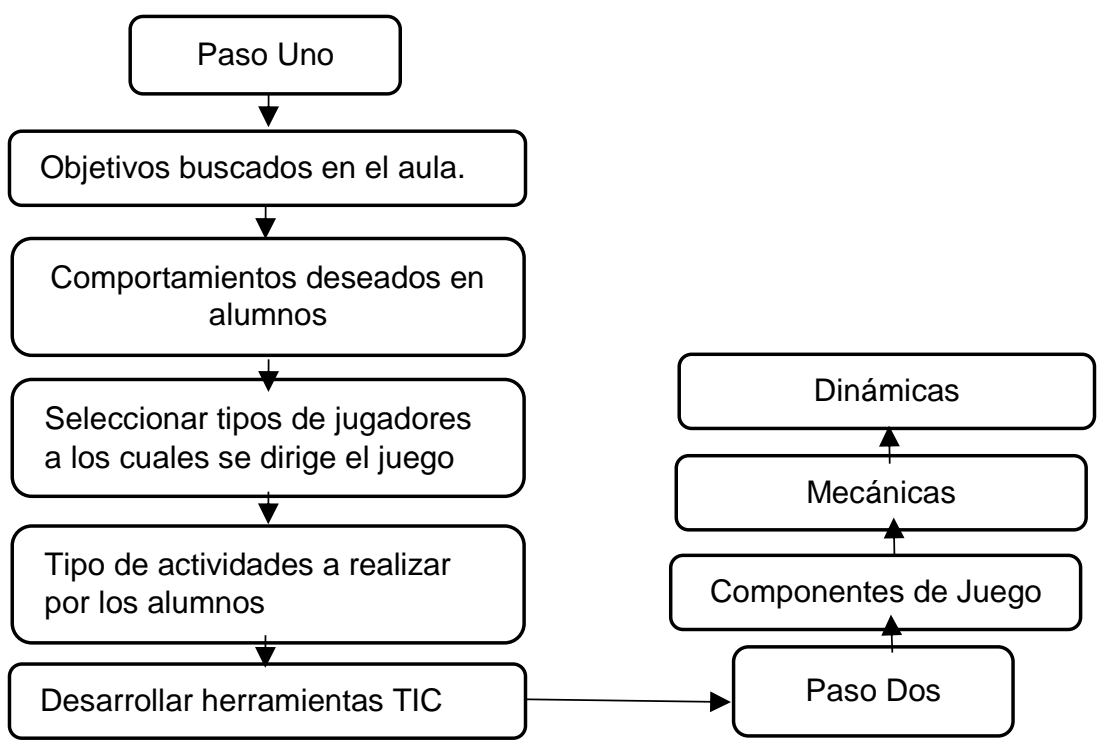

Fig. 1: Adaptación de la metodología 6D de Werbach y Hunter 2012

En el paso número uno, se llevó a cabo la definición de las primeras cinco etapas de la metodología de Werbach y Hunter, el paso "Incluir la diversión" se omitió debido a la complejidad del término. Cada una de estas cinco etapas obtuvo resultados respecto a las labores de diseño de juego, los resultados de las definiciones de cada una de estas etapas se plasmaron en un diagrama.

Los resultados correspondientes a las primeras tres etapas se muestran en la Fig. 2.

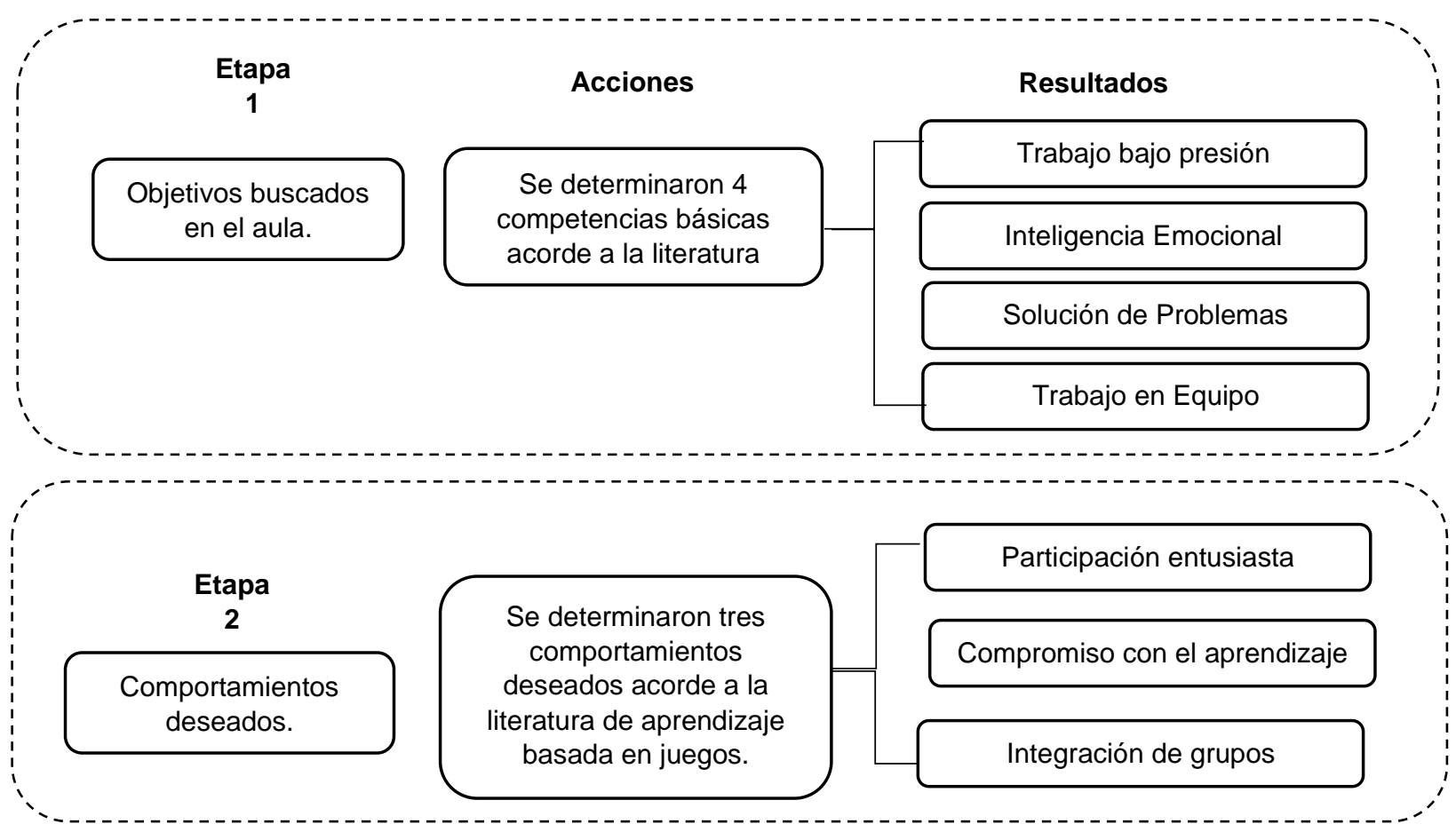

Fig. 2: Etapas iniciales de labores de diseño de juego

Al finalizar la ejecución de las primeras dos etapas las cuales son esenciales para definir los objetivos del juego, se prosiguió a la ejecución de las tres etapas posteriores donde se especifican los medios para cumplir los objetivos previamente plasmados, dichos medios arrojaron los resultados presentados en la Fig. 3.

Los resultados que se muestran en la Fig. 3 son esenciales para la cumplir los objetivos plasmados en las etapas mostradas en la Fig. 2. Al finalizar las etapas de diseño de juego se llevó a cabo la ejecución del paso dos (ver Fig. 1), en dicho proceso se realizó la selección de los elementos de juego primarios y secundarios, 
los cuales se integraron a la propuesta; tanto los resultados obtenidos en el paso uno (objetivos y orientación del juego) como los del paso dos (elementos del juego), fueron agrupados. Dicha agrupación de resultado se presenta en forma de propuesta en la siguiente sección.

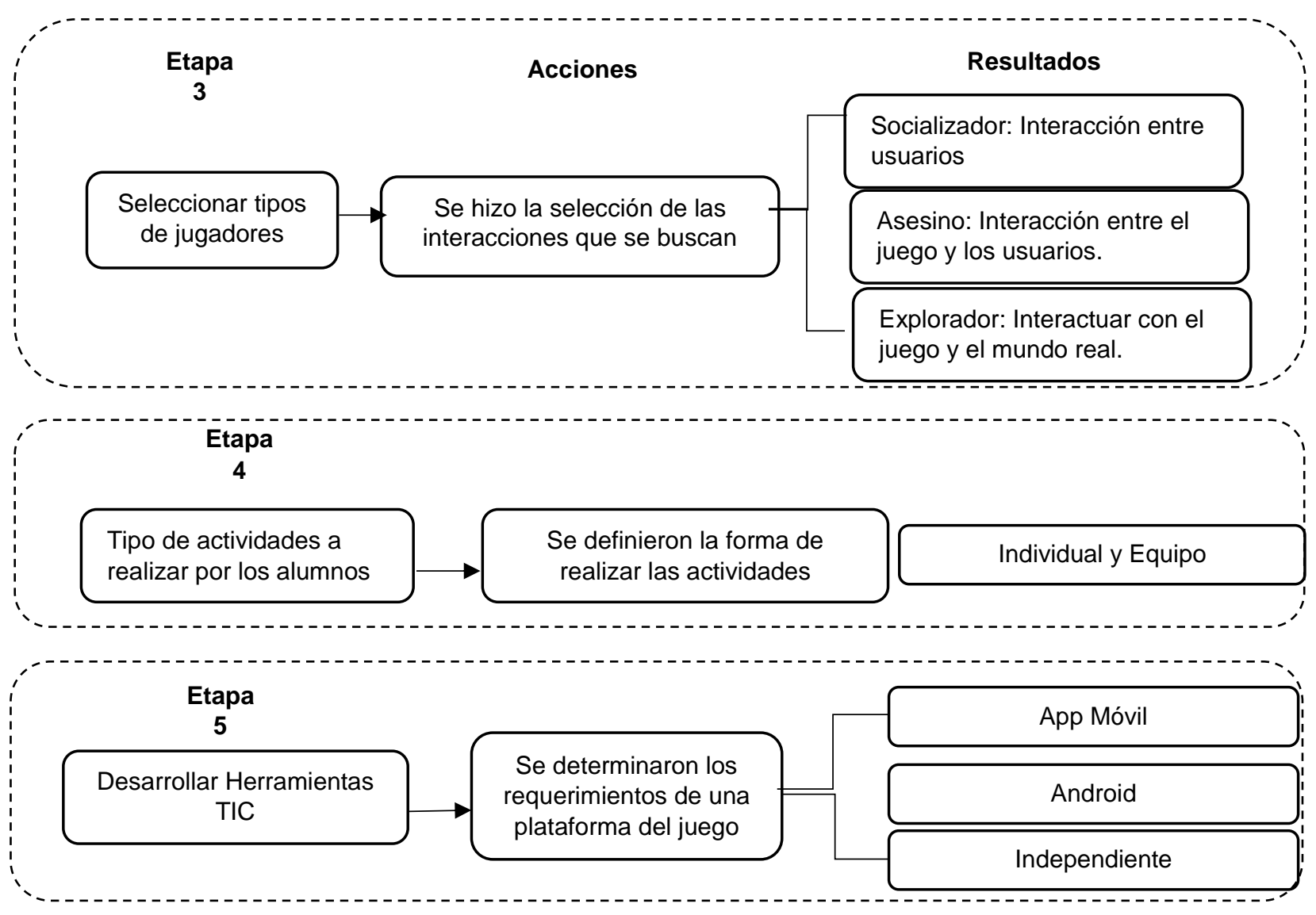

Fig. 3: Etapas finales de labores de diseño de juego

\section{RESULTADOS}

Al finalizar los dos pasos de la metodología adaptada, y como resultado del análisis de los resultados obtenidos; se obtuvieron dos diagramas, los cuales fungen como base teórica y esquemática para la elaboración de la propuesta del juego. Es decir, se obtuvo una propuesta, la cual integra: 1) Objetivos en el aula, 3) Comportamientos deseados y 4) Elementos de juego. La propuesta se presenta en forma de diagramas, los cuales permiten facilitar la comprensión y la retención de las dinámicas. Así mismo es necesario enfatizar que cada diagrama funciona como guía para comenzar con las labores de programación del juego.

El primer diagrama es un esquema del funcionamiento los componentes primarios de juego, es decir, el propósito de este diagrama es poner las reglas bajo las cuales funcionaran dichos componentes. La importancia de dejar en claro el funcionamiento de los componentes primarios es debido a que estos son herramientas fundamentales para la construcción del entorno del aprendizaje basado en juegos (ver Fig. 4). Como se puede apreciar en la Fig. 4 los componentes principales del juego, son misiones, puntos, y cuentas regresivas, esto debido a que este tipo de actividades fomentan la solución de problemas, el trabajo bajo presión y la inteligencia emocional. Así mismo la Fig. 4 aclara las condiciones previas necesarias para dar inicio al juego las cuales son: 1) Reclutar y juntar un mínimo de 15 jugadores, y 2) Diseñar al menos 12 misiones a desarrollar durante el juego, las cuales especifiquen detalladamente la actividad a realizar, si debe ser realizada en grupo o de manera individual, los puntos de recompensa o penalización que se pueden obtener, así como el tiempo límite para llevar a cabo la tarea.

En la Fig. 4 se hace énfasis en las misiones motivo por el cual en este apartado de explica de manera un poco más amplia el funcionamiento y objetivo de dichas misiones. Las misiones serán enviadas por el docente a cargo del juego a través de la aplicación móvil, el docente puede diseñar un máximo de 60 misiones para 
enviar a los alumnos. Así mismo el alumno es libre de aceptar o declinar la misión, acorde a lo que considere pertinente para su desempeño y su aprendizaje.

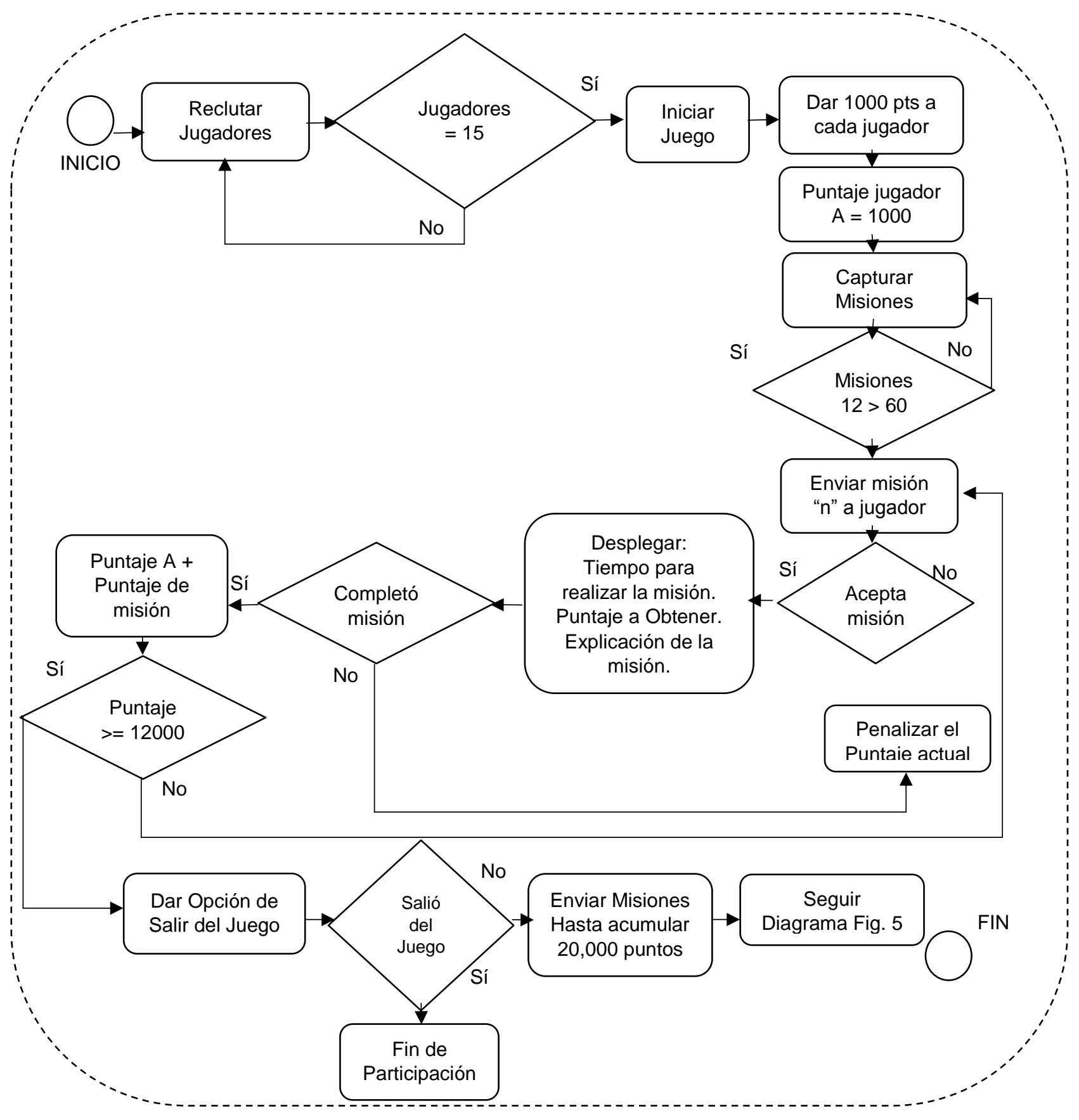

Fig. 4: Componentes Primarios del Juego

La importancia de las misiones radica en que permiten al alumno evaluar (observación), vivir (aprendizaje basado en problemas), tomar decisiones en tiempo real (Simulación), acerca de un problema en específico; es decir, las misiones combinan algunas de las técnicas de generación y evaluación de competencias, motivo por el cual las misiones son un punto clave en esta propuesta. Sin embargo, las misiones y los puntos no son todos los componentes que juego integra, también se deben integrar puntajes, niveles y tablas de calificación, como componentes de juego secundarios, para fomentar el compromiso, la participación y la competitividad de los alumnos al enfrentarlos ante la evaluación constante de su desempeño en el juego, dichos componentes se presentan en forma de diagrama (ver Fig. 5).

El diagrama presentado en la Fig. 5 agrupa los componentes secundarios del juego es decir las mecánicas y dinámicas diseñadas para cumplir tanto con los comportamientos deseados en el alumnado como con los objetivos buscados en el aula. Así mismo muestra el funcionamiento de dichos componentes, y también muestra los requisitos necesarios para finalizar el juego. 


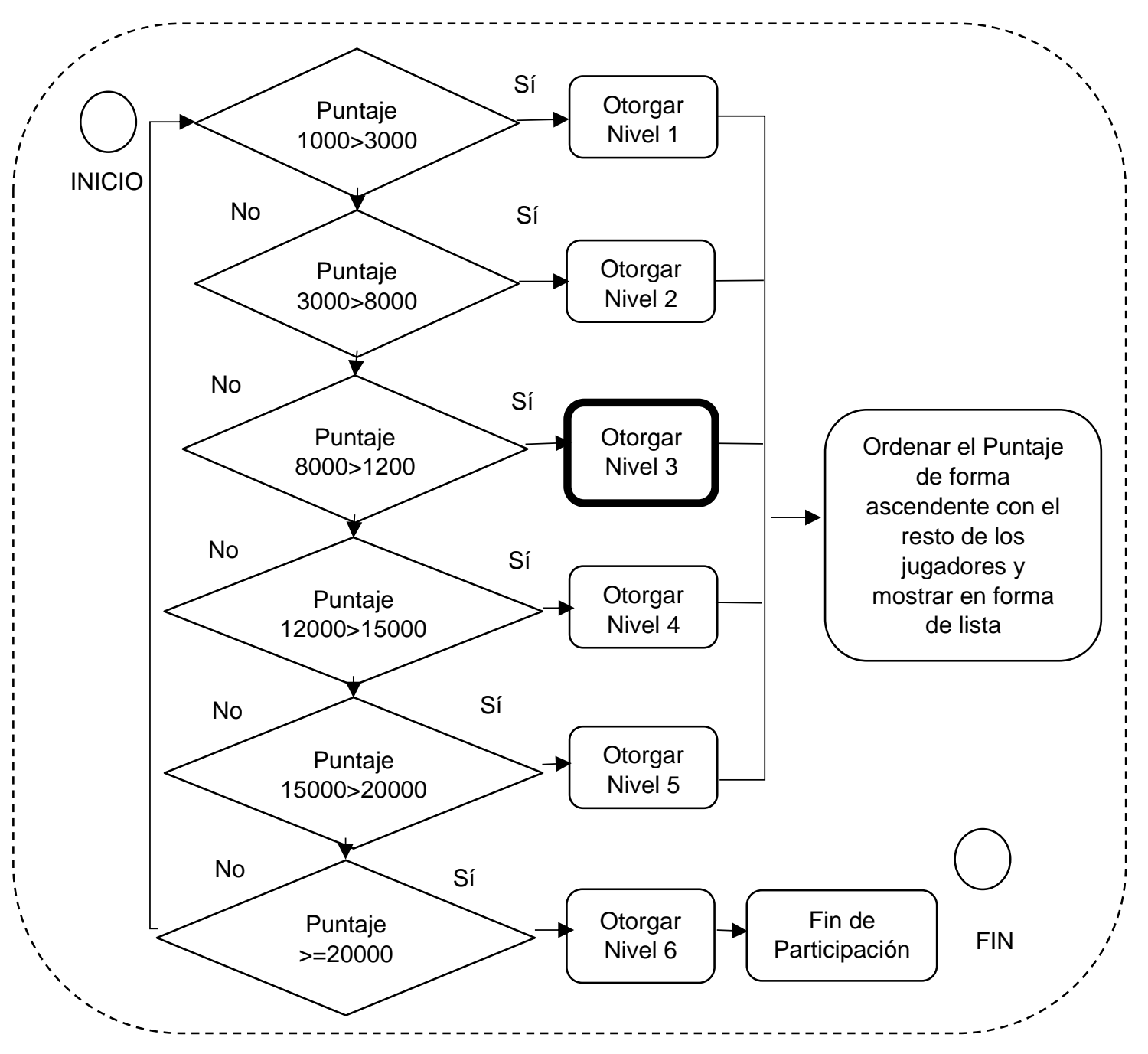

Fig. 5: Componentes de Juego secundarios

Dentro del juego se contemplan cinco niveles cuya dificultad va cambiando al igual que las recompensas obtenidas por misiones. Para que un alumno pueda aprobar el juego con calificación mínima de seis, el alumno tiene que alcanzar el nivel 3 , en este nivel el alumno tiene la oportunidad de continuar o abandonar el juego. $\mathrm{Si}$ el alumno decide continuar en el juego tiene la posibilidad de incrementar tanto su calificación como su nivel. El nivel será visible todo el tiempo para el alumno, el nivel será asignado acorde al puntaje que cada alumno obtenga (ver Fig. 5).

Cuando el nivel sea asignado y el puntaje total calculado, se proseguirá a ordenar el puntaje de forma ascendente de todos los jugadores y se creara una tabla de puntuaciones, la cual se mostrará en forma de lista a todos los jugadores. Independientemente de los componentes primarios y secundarios descritos con anterioridad; también se contemplan de manera intrínseca dos componentes extras de juego para reforzar el compromiso de los alumnos 1) Libre elección: Este componente está presente en todo el juego ya que el alumno sabrá que decisiones tomar, que retos aceptar, y tendrá la opción de continuar o no en el juego 2) Retroalimentación: Los alumnos recibirán una retroalimentación constructiva de su desempeño en forma de puntos

La estructura de la propuesta teórica de juego presentada en la Fige. 4 y en la Fig. 5, es una fase inicial para el desarrollo de un juego en Android que funcione de manera independiente, la finalidad de que esta propuesta se ejecute en una plataforma Android es incluir las TIC en el proceso de aprendizaje; el uso de las TIC independientemente de ser un fuerte motivador para los estudiantes, funciona como una herramienta fundamental para la implementación del juego y ambas son infalibles para poder incorporar la Gamificación en el proceso de aprendizaje y evaluación dentro del aula.

La Gamificación es una herramienta que se ha posicionado desde su incursión en el buscador de google en el año 2008, y se ha aplicado en varios niveles educativos obteniendo resultados satisfactorios en la retención y en las calificaciones finales de los participantes (Brull y Finlayson, 2016; Admiraal et al., 2014). En 
universidades el aprendizaje basado en juegos también ha sido aplicado, en ingeniería civil para el aprendizaje de estructuras (Ebner y Holzinger, 2007), también se ha incluido en cursos de tecnologías de información y comunicación, con la finalidad de motivar intrínseca y extrínsecamente a los estudiantes a reforzar más los conceptos vistos en clase (Çakıroğlu et al., 2017); si bien la rama de la ingeniería y la de ciencias de la computación han hecho uso de los beneficios de la Gamificación, las ciencias sociales no se han quedado atrás, en esta rama se han valido de esta técnica para reforzar contenido teórico de algunos tópicos particularmente pesado en clases tales como Filosofía, Administración y Operaciones (Papastergiou, 2009). Todas estas investigaciones han tenido resultados satisfactorios y medibles en el desempeño académico de los estudiantes y en su compromiso con las asignaturas.

Como futuras investigaciones se contempla el uso de la aplicación, y la evaluación tanto del sistema como de la interfaz por parte de los profesores y alumnos, con la finalidad de definir si existe una relación entre los logros de los alumnos con los elementos de Gamificación. Una vez puesta en marcha esta primera prueba, es importante dar seguimiento al juego de manera continua, y así comprobar la efectividad de la Gamificación de manera cuantitativa.

\section{CONCLUSIONES}

Esta propuesta de juego posee como ventaja comparativa al resto de los juegos diseñados para universidades que: busca el desarrollo de competencias de los alumnos, lo cual lo califica a la propuesta como replicable y viable para toda aquella universidad que busque el desarrollar competencias en sus alumnos a través de los elementos del aprendizaje basado en juegos. La programación del juego actualmente se encuentra en fase inicial, la cual está lista para ser implementada en cursos próximos.

Esta investigación a diferencia del resto de juegos diseñados para universidades, tiene el objetivo particular de apoyar al desarrollo de competencias en los estudiantes motivo por el cual el juego tiene como base para su evaluación mecánicas empleadas en la evaluación por competencias, estas mecánicas de evaluación fueron aquellas sugeridas por Moreno. Este juego está diseñado para fungir como herramienta multidisciplinaria complementaria a la enseñanza, es decir la propuesta de juego aquí presentada tiene la particularidad de que puede adaptarse a cualquier rama de la ciencia y a las demandas de cada profesor en específico, sin dejar de lado en desarrollo de competencias básicas.

\section{AGRADECIMIENTOS}

Agradecemos las facilidades otorgadas para el desarrollo del presente trabajo al Instituto Politécnico NacionalUnidad Profesional Interdisciplinaria de Ingeniería y Ciencias Sociales y Administrativas. Proyecto SIP 20161046 Las Habilidades Informáticas Del Capital Humano Como Fuente De Crecimiento Económico.

\section{REFERENCIAS}

Admiraal, W., J. Huizenga y otros cuatro autores, Gender-inclusive game-based learning in secondary education, doi: 10.1080/13603116.2014, Int. J. Incl. Educ, 18(11), 1208-1218 (2014)

Aldemir, T., B. Celik y G. Kaplan, A qualitative investigation of student perceptions of game elements in a gamified course, doi: 10.1016/J.CHB.2017.10.001, Comput. Hum. Behav., 78, 235-254 (2018)

Alhammad, M.M. y A.M. Moreno, Gamification in software engineering education: A systematic mapping, doi: 10.1016/J.JSS.2018.03.065, J. Syst. Software, 141, 131-150 (2018)

Amriani, A., A. F. Aji, A. Y. Utomo y K.M. Junus, An empirical study of gamification impact on e-Learning environment, doi: 10.1080/13603116.2014.885592, In Computer Science and Network Technology (ICCSNT), 3rd International Conference, 265-269 China (2013)

Brull, S. y S. Finlayson, Importance of Gamification in Increasing Learning, doi: 10.3928/00220124-20160715-09, J. Contin. Educ. Nursing, 47(8), 372-375 (2016)

Çakıroğlu, Ü, B. Başıbüyük y otros tres autores, Gamifying an ICT course: Influences on engagement and academic performance, doi: 10.1016/J.CHB.2016.12.018, Comput. Hum Behav., 69, 98-107 (2017)

Chu, C. y C. H. Hung, Effects of the Digital Game-Development Approach on Elementary School Students' Learning Motivation, Problem Solving, and Learning Achievement, doi: 10.4018/ijdet.2015010105, International Journal of Distance Education Technologies (IJDET), 13 (1), 87-102 (2015)

Consejo Mexicano de Investigación Educativa y T. Moreno, Reseña de "Educar por Competencias ¿Qué hay de Nuevo?" ISSN: 1405-6666, Revista Mexicana de Investigación Educativa, 15 (44), 289-297 (2015)

Deterding, S., D. Dixon, R. Khaled y L. Nacke, From Game Design Elements to Gamefulness: Defining Gamification, doi: $10.1145 / 2181037.2181040$, Proceedings of the 15th international academic MindTrek conference: Envisioning future media environments, ACM, 11(1), 9-15 (2011) 
Deterding, S., M. Sicart y otros tres autores, Gamification. Using Game-Design Elements in Non-Gaming Contexts, doi: 10.1145/1979742.1979575, CHI'11 Extended Abstracts on Human Factors in Computing Systems, ACM, 2425-2428 (2011)

Ebner, M. y A. Holzinger, Successful implementation of user-centered game based learning in higher education: An example from civil engineering, doi: 10.1016/J.COMPEDU.2005.11.026, Comput. Educ., 49(3), 873-890 (2007)

García, A., V. Basilotta y C. López, Las TIC en el aprendizaje colaborativo en el aula de Primaria y Secundaria, doi: 10.3916/C42-2014-06, Comunicar, 21(42), 65-74 (2014)

Gimeno, J., Educar por competencias: ¿Qué hay de Nuevo? 1ª Ed., 236, Ediciones Morata, Madrid, España (2008)

Khan, A., F. Ahmad y M. Malik, Use of digital game based learning and Gamification in secondary school science, doi: 10.1007/s10639-017-9622-1, The effect on student engagement, learning and gender difference, Educ. Inf. Technol., 22(6), 2767-2804 (2017)

Landers, R.N., M. B. Armstrong y A.B. Collmus, How to Use Game Elements to Enhance Learning: Applications of the Theory of Gamified Learning, doi: 10.1007/978-3-319-51645-5_21, In: Ma M., Oikonomou A. (Eds.) Serious Games and Edutainment Applications, Springer Cham, 457-483 (2017)

Lee, H. e Y.Y. Doh, A Study on the Relationship between Educational Achievement and Emotional Engagement in a Gameful Interface for Video Lecture Systems, doi: 10.1109/ISUVR.2012.21, International Symposium on Ubiquitous Virtual Reality, 1 (1), 34-37 (2012)

López, C., V. Benedito y M. J. León, El Enfoque de Competencias en la Formación Universitaria y su Impacto en la Evaluación, la Perspectiva de un Grupo de Profesionales Expertos en Pedagogía, doi: 10.4067/S071850062016000400003, Formación Universitaria, 9(4), 11-22 (2016)

Moreno, T., La evaluación de competencias en educación, ISSN: 2007-7033, Sinéctica, 39 (1), 01-20 (2012)

Papastergiou, M., Digital Game-Based Learning in high school Computer Science education: Impact on educational effectiveness and student motivation doi: 10.1016/j.compedu.2008.06.004, Comput. Educ., 52 (1), 1-12 (2009)

Robson, K., K. Plangger y otros tres autores, Understanding Gamification of Consumer Experiences, https://goo.gl/fn3WZK, Advances in Consumer Research, 42(2), 352-356 (2015)

Sanmugam, M., Z. Abdullah y otros cuatro autores, The Affiliation between Student Achievement and Elements of Gamification in Learning Science, doi: 10.1109/ICoICT.2016.7571962, 4th International Conference on Information and Communication Technology (ICoICT), 1-4 (2016)

Werbach, K. y D. Hunter, For the Win: How Game Thinking Can Revolutionize Your Business, 1ts Edition, 147, Wharton Digital Press, Philadelphia-Pennsylvania (2012)

Zichermann, G. y C. Cunningham, Gamification by Design: Implementing Game Mechanics in Web and Mobile Apps, 1ts Edition, 180-182, O'Reilly Media, Inc. California-USA (2011)

Zichermann, G. y J. Linder, Game-Based Marketing: Inspire Customer Loyalty through Rewards, Challenges and Contests, 1ts Edition, 240, John Wiley and Sons, New Jersey-USA (2010) 
